\title{
ELECTRON TEMPERATURES OF PLANETARY NEBULAE DETERMINED FROM THE He I DISCONTINUITIES
}

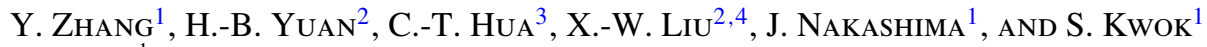 \\ ${ }^{1}$ Department of Physics, University of Hong Kong, Hong Kong; zhangy96@ hku.hk \\ ${ }^{2}$ Department of Astronomy, Peking University, Beijing 100871, China \\ ${ }^{3}$ Observatoire Astronomique de Marseille-Provence Laboratoire d'Astrophysique de Marseille Pôle de l'Etoile Site de Château-Gombert 38, \\ rue Frédéric Joliot-Curie 13388, Marseille Cedex 13, France \\ ${ }^{4}$ Kavli Institute for Astronomy and Astrophysics, Peking University, Beijing 100871, China \\ Received 2008 October 23; accepted 2009 January 13; published 2009 March 31
}

\begin{abstract}
We have used the He I discontinuities at $3421 \AA$ to determine the electron temperatures, designated $T_{\mathrm{e}}(\mathrm{He} \mathrm{I})$, for a sample of five Galactic planetary nebulae $(\mathrm{PNe})$. We compared $T_{\mathrm{e}}(\mathrm{He} \mathrm{I})$ with the electron temperatures derived from the hydrogen Balmer jump at $3646 \AA$, designated $T_{\mathrm{e}}(\mathrm{H} \mathrm{I})$, and found that $T_{\mathrm{e}}(\mathrm{He} \mathrm{I})$ are generally lower than $T_{\mathrm{e}}(\mathrm{H} \mathrm{I})$. There are two possible interpretations, (1) the presence of substantial $\mathrm{He}^{2+}$ zone, or (2) the presence of hydrogen-deficient cold clumps within diffuse nebulae. A series of photoionization models were constructed to test the two scenarios. We found that the observed $T_{\mathrm{e}}(\mathrm{He} \mathrm{I}) / T_{\mathrm{e}}(\mathrm{H} \mathrm{I})$ discrepancies are beyond the predictions of chemically homogeneous models. Our modeling shows that the presence of a small amount of hydrogen-deficient inclusions seems to be able to reproduce the observed intensities of He I discontinuities. We stress the value of $\mathrm{He}$ I discontinuities in investigating nebular physical conditions. Albeit with some observational and technical limitations, He I discontinuities should be considered in future modeling work.
\end{abstract}

Key words: ISM: general - planetary nebulae: general

\section{INTRODUCTION}

The accurate determination of chemical abundances of planetary nebulae $(\mathrm{PNe})$ is of fundamental importance to the understanding of the nucleosynthesis of low- and intermediate-mass stars and the chemical evolution of galaxies. However, one of the main problems in nebular astrophysics is that the heavy element abundances derived from collisionally excited lines (CEL) are often lower than those derived from optical recombination lines (ORLs). The typical ORL/CEL abundance discrepancy factor (ADF) is $\sim 2$ for general PNe. The most extreme case to date is Hf 2-2, which has an ADF of about 70 (Liu et al. 2006). Recent reviews on this problem have been presented by Liu (2006) and Peimbert \& Peimbert (2006; see also the references therein). There are two possible solutions: (1) the presence of temperature and density variations in chemically homogeneous nebulae; (2) the two-component nebular model with hydrogen-deficient inclusions embedded in the diffuse nebula. In order to test the two scenarios, we need to further investigate nebular physical conditions, in particular for the regions that ORLs originate in. For this purpose, new plasma diagnostic tools rather than the classical CEL diagnostics are badly required.

Zhang et al. (2004) applied the hydrogen Balmer jump to determine the electron temperatures-hereafter $T_{\mathrm{e}}(\mathrm{H} \mathrm{I})$-for a large sample of PNe. They found that $T_{\mathrm{e}}(\mathrm{H} \mathrm{I})$ is systematically lower than that derived from the ratio of nebular to auroral lines of [O III] -hereafter $T_{\mathrm{e}}(\mathrm{O}$ III $)$, consistent with previous results by Liu \& Danziger (1993). The discrepancies between $T_{\mathrm{e}}(\mathrm{H} \mathrm{I})$ and $T_{\mathrm{e}}(\mathrm{O}$ III $)$ were first studied by Peimbert $(1967,1971)$, who found that temperature variations within nebulae may lead to higher $T_{\mathrm{e}}(\mathrm{O}$ III $)$ compared to $T_{\mathrm{e}}(\mathrm{H} \mathrm{I})$. To quantitatively study the problem, they defined the mean square temperature variation, $t^{2}$. Photoionization models of chemically and spatially homogeneous nebulae yielded typical values of $t^{2}$ between 0.003 and 0.015 (see Peimbert \& Peimbert 2006). However, the observations by Liu \& Danziger (1993) and Zhang et al. (2004) indicated considerably large temperature variations, which are far beyond the predictions of typical photoionization models. This can be ascribed to either additional energy inputs for chemically homogeneous nebulae or chemical inhomogeneities.

Zhang et al. (2005a, 2005b) developed a method to use the $\mathrm{He}$ I recombination line ratios to diagnose electron temperatureshereafter $T_{\mathrm{e}}(\mathrm{He} \mathrm{I})$. The comparison between $T_{\mathrm{e}}(\mathrm{He} \mathrm{I})$ and $T_{\mathrm{e}}(\mathrm{H} \mathrm{I})$ is essential to discriminate between the two-abundance model and chemically homogeneous nebulae with temperature and density variations as responsible for the CEL/ORL abundance problem since the two scenarios predict different relations between $T_{\mathrm{e}}(\mathrm{He} \mathrm{I})$ and $T_{\mathrm{e}}(\mathrm{H} \mathrm{I})$. From a study of a sample of $48 \mathrm{PNe}$, Zhang et al. (2005a; hereafter Paper I) found that $T_{\mathrm{e}}(\mathrm{He} \mathrm{I})$ is significantly lower than $T_{\mathrm{e}}(\mathrm{H} \mathrm{I})$, in favor of the twoabundance model. However, there are two arguable problems. Peimbert \& Peimbert (2006) found that for some PNe the $T_{\mathrm{e}}(\mathrm{He} \mathrm{I})$ values derived from the $\mathrm{He} \mathrm{I} \lambda \lambda 3889,4471$, and 7069 are different with those derived from the He I $\lambda \lambda 6678,7281$. This might be partially caused by the uncertainties of the density of $\mathrm{He}^{+}$zones and/or the optical depth effects on the He I 23889 . Peimbert \& Peimbert (2006) proposed that at least 10 different $\mathrm{He}$ I lines are required to accurately deduce these parameters. Moreover, for high-excitation $\mathrm{PNe}$, the $\mathrm{He}^{+}$and $\mathrm{H}^{+}$zones are not identical, and the presence of a substantial $\mathrm{He}^{2+}$ zone may partially contribute to the difference between $T_{\mathrm{e}}(\mathrm{He} \mathrm{I})$ and $T_{\mathrm{e}}(\mathrm{H} \mathrm{I})$.

These methods have also been used to determine the electron temperatures of H II regions (García-Rojas et al. 2005; Guseva et al. 2006, 2007). These authors found that the temperature differences in $\mathrm{H}$ II regions are not so obvious as those found in $\mathrm{PNe}$.

In this paper, we use an alternative method, fitting the $\mathrm{He} \mathrm{I}$ discontinuities, to determine $T_{\mathrm{e}}(\mathrm{He} \mathrm{I})$ for five Galactic PNe. This method is made possible by the availability of high signal-to-noise spectra of these nebulae, resulting in a clear detection of the helium jump. The current study allows us to 
Table 1

$T_{\mathrm{e}}(\mathrm{He} \mathrm{I})$ and $T_{\mathrm{e}}(\mathrm{H} \mathrm{I})$

\begin{tabular}{|c|c|c|c|c|c|}
\hline \multirow[t]{2}{*}{ Source } & \multirow[t]{2}{*}{$\mathrm{He}^{2+} / \mathrm{He}^{+\mathrm{a}}$} & \multicolumn{2}{|c|}{$T_{\mathrm{e}}\left(\mathrm{He} \mathrm{I}^{\mathrm{b}}(\mathrm{K})\right.$} & \multicolumn{2}{|c|}{$T_{\mathrm{e}}\left(\mathrm{H}_{\mathrm{I}}\right)(\mathrm{K})$} \\
\hline & & This Work & Paper I & This Work & Paper I \\
\hline IC 2501 & 0.002 & $4700 \pm 3200$ & & $10000 \pm 600$ & . \\
\hline IC 4634 & 0.000 & $3800 \pm 2800$ & $5000 \pm 1000$ & $10000 \pm 500$ & $8500 \pm 400$ \\
\hline NGC 2440 & 0.401 & $5700 \pm 4100^{c}$ & $\ldots$ & $15000 \pm 800$ & $\ldots$ \\
\hline NGC 3242 & 0.230 & $5000 \pm 2800^{\mathrm{d}}$ & $4500 \pm 1000$ & $12000 \pm 1200$ & $10200 \pm 1000$ \\
\hline NGC 3918 & 0.303 & $5500 \pm 3900^{\mathrm{e}}$ & $6000 \pm 1000$ & $11000 \pm 300$ & $12300 \pm 1000$ \\
\hline
\end{tabular}

Notes.

${ }^{a}$ From Cahn et al. (1992).

${ }^{\mathrm{b}}$ Note that the $T_{\mathrm{e}}(\mathrm{He} \mathrm{I})$ values given in Paper I are derived from He I line ratios.

${ }^{\mathrm{c}}$ If merely the spectral range from $3300 \AA$ to $3600 \AA$ was taken into account, we derived a value of $6840 \mathrm{~K}$, which represents the upper limit of $T_{\mathrm{e}}(\mathrm{He} \mathrm{I})$ (see the dotted lines in the right panel of Figure 2).

$\mathrm{d}$ The upper limit is $7500 \mathrm{~K}$. Details are as indicated in footnote $\mathrm{c}$.

e The upper limit is $8250 \mathrm{~K}$. Details are as indicated in footnote c.

further investigate the relation between $T_{\mathrm{e}}(\mathrm{He} \mathrm{I})$ and $T_{\mathrm{e}}(\mathrm{H} \mathrm{I})$. We examine the two-abundance models and photoionization models of chemically homogeneous nebulae as the explanation of the observed $T_{\mathrm{e}}(\mathrm{H} \mathrm{I}) / T_{\mathrm{e}}(\mathrm{He} \mathrm{I})$ discrepancies. This paper is a complement to the authors' work on recombination spectra as probes of physical conditions of PNe (Liu \& Danziger 1993; Zhang et al. 2004, 2005a, 2005b).

\section{OBSERVATIONS AND DATA REDUCTION}

The sample includes two low-excitation PNe and three highexcitation PNe (see Table 1). The long-slit spectra were obtained using the f-18 Nasmyth focus of the $2.3 \mathrm{~m}$ Advanced Technology Telescope at Siding Spring Observatory on 2000 March. Both arms of the Double Beam Spectrograph (DBS) were used with the 1200B grating in the second order to give spectral resolution of $0.3 \AA$ pixel $^{-1}$. The dichroic may be removed or replaced with a flat mirror allowing the use of either arm independently. The detector was a SITE $1752 \times 532$ chip, giving a spectral coverage from 3240 to $8520 \AA$. In order to avoid loss of light due to atmospheric refraction each PN was observed with the spectrograph slit aligned to the parallactic angle. Furthermore, all the observations were carried out under the best seeing conditions, along with airmass around 1.2 to ensure the total UV light into the spectrograph slit. For each PN, the exposure time was $1500 \mathrm{~s}$.

The LONG92 package in MIDAs ${ }^{5}$ was used for the data reduction following the standard steps, including bias subtraction, flat-field correction, cosmic-ray rejection, and wavelength calibration with a helium-argon calibration lamp. Flux calibration was conducted using the spectroscopic standards EG 131 and LTT 4364. Finally, the spectra were dereddened using the standard Galactic extinction law for a total-to-selective extinction ratio of $R=3.1$ (Howarth 1983) and the extinction coefficients given by Cahn et al. (1992).

\section{ANALYSIS AND RESULTS}

The spectra allow us to simultaneously determine $T_{\mathrm{e}}(\mathrm{He} \mathrm{I})$ and $T_{\mathrm{e}}(\mathrm{H} \mathrm{I})$ in terms of the $\mathrm{He} \mathrm{I}$ discontinuities at $3421 \AA$ $\left(J_{\mathrm{He}}=I_{3421^{-}}-I_{3421^{+}}\right)$, produced by $\mathrm{He}^{+}$recombination to the He I $2 p^{3} P^{\circ}$ level, and the $\mathrm{H}$ I Balmer discontinuities at $3646 \AA$ $\left(J_{\mathrm{H}}=I_{3646^{-}}-I_{3646^{+}}\right)$. Note that we do not distinguish between the electron temperatures derived from the $\mathrm{He}$ I discontinuities

5 MIDAS is developed and distributed by the European Southern Observatory.

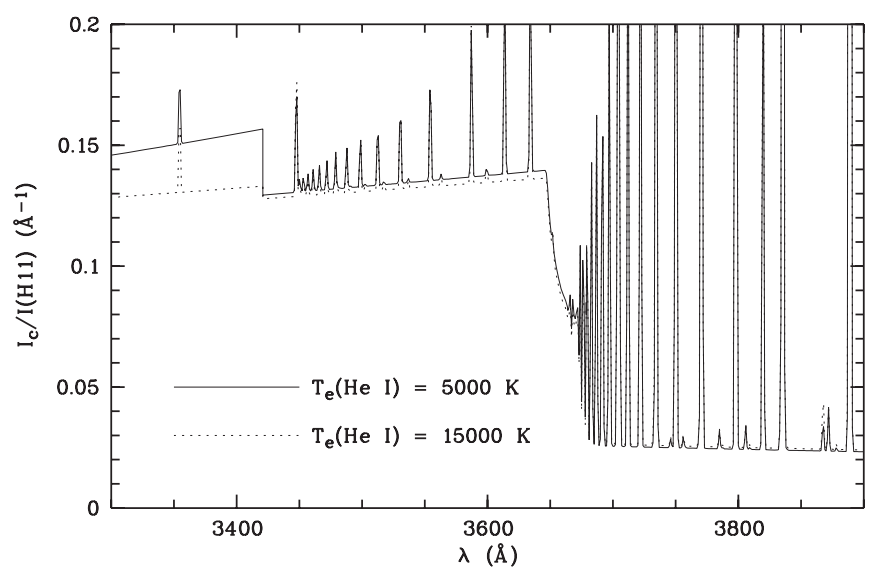

Figure 1. Theoretical $\mathrm{H}$ I and $\mathrm{He}$ I recombination spectra at different $T_{\mathrm{e}}(\mathrm{He} \mathrm{I})$. For the calculations, we have assumed $T_{\mathrm{e}}(\mathrm{H} \mathrm{I})=10,000 \mathrm{~K}, \mathrm{He}^{+} / \mathrm{H}^{+}=0.1$, and $\mathrm{He}^{2+} / \mathrm{He}^{+}=0$.

and from the $\mathrm{He} \mathrm{I}$ lines and use $T_{\mathrm{e}}(\mathrm{He} \mathrm{I})$ to denote both. The method is similar to that used to obtain $T_{\mathrm{e}}(\mathrm{H} \mathrm{I})$ in Zhang et al. (2004). To synthesize the spectra, we consider the contributions from the free-free, free-bound, and bound-bound emission from $\mathrm{H} \mathrm{I}, \mathrm{He}$ I, and $\mathrm{He}$ II and the two-photon decay from the $2^{2} S$ level of hydrogen. The coefficients of the H I, He I, and $\mathrm{He}$ II continuous emission as functions of temperature are available in Ercolano \& Storey (2006). The scattering from the central star is simulated using a modified blackbody function, $I_{\lambda} \propto \lambda^{-\beta} B(\lambda, T)$, where $\beta$ is the spectral index $(\beta=0$ for standard blackbody spectrum), $B(\lambda, T)$ is the Planck function, and $T$ is the temperature of the central star. We refer the reader to Zhang et al. (2004) for a detailed description of our fitting procedure. In order to illustrate our method, Figure 1 gives the theoretical spectra at different $T_{\mathrm{e}}(\mathrm{He} \mathrm{I})$. It is clear that increasing of $T_{\mathrm{e}}(\mathrm{He} \mathrm{I})$ will decrease the $\mathrm{He} \mathrm{I}$ discontinuities. This is a consequence of the fact that the number of the lowestenergy free electrons, which is proportional to the intensity of the He I free-bound emission at $3421^{-} \AA$, decreases with increasing electron temperature. For the same reason, the $\mathrm{H}$ I Balmer discontinuities decrease with increasing $T_{\mathrm{e}}(\mathrm{H} \mathrm{I})$. Note that the $J_{\mathrm{H}}$ values may slightly change with $T_{\mathrm{e}}(\mathrm{He} \mathrm{I})$ because of a small contribution from the $\mathrm{He}$ I discontinuity at $3680 \AA$ A produced by recombination to the $2{ }^{1} P$ level.

Consequently, $T_{\mathrm{e}}(\mathrm{He} \mathrm{I})$ and $T_{\mathrm{e}}(\mathrm{H} \mathrm{I})$ can be derived by fitting the theoretical to the observed spectra. Before the fitting, we 

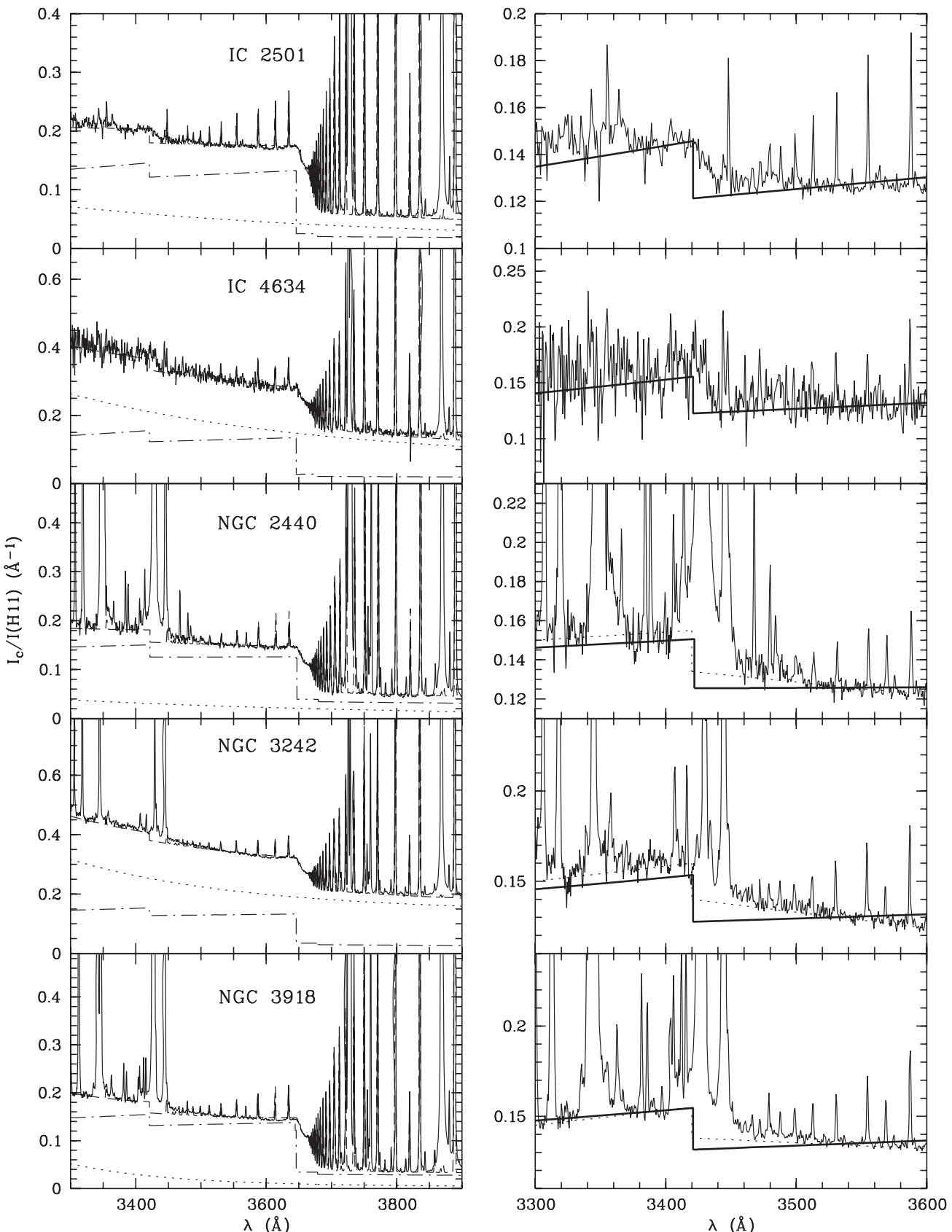

Figure 2. Left panels: a comparison of observed (solid lines) and theoretical (dashed lines) spectra. The dotted-dashed lines and the dotted lines represent the theoretical nebular continuum and scattered stellar light, respectively. Right panels: zoom-in views of the spectra around the He I discontinuities at 3421 A. The contributions from scattered-light continuum have been subtracted. The modeling nebular continuities are overlaid with thick solid lines. Note that although our models give the best fit to the global spectra from $3240 \AA$ to $4000 \AA$, they may not satisfactorily reproduce the narrow spectral ranges of the three high-excitation PNe from $3421 \AA$ to $3500 \AA$ for a variety of reasons (see the text). The dotted lines denote the best fit to the narrow spectral ranges.

require to measure the $\mathrm{He}^{+} / \mathrm{H}^{+}$and $\mathrm{He}^{2+} / \mathrm{He}^{+}$abundance ratios. The $\mathrm{He}^{+} / \mathrm{H}^{+}$ratios are obtained from a few strong $\mathrm{He}$ I lines around the $\mathrm{He}$ I discontinuities. The deduced $\mathrm{He}^{+} / \mathrm{H}^{+}$ratios are relatively insensitive to the adopted electron temperatures and densities. The flux uncertainties of the He I lines are less than $15 \%$. The $\mathrm{He}^{2+} / \mathrm{He}^{+}$ratios are taken from Cahn et al. (1992) (see Table 1). The errors in temperature determination caused by uncertainties of the $\mathrm{He}^{2+} / \mathrm{He}^{+}$ratios are negligible since the contribution from $\mathrm{He}$ II to the continuum emission is much smaller than that from $\mathrm{H}$ I and $\mathrm{He} \mathrm{I}$.

Figure 2 shows the fitting results for the five PNe. In order to reduce the errors caused by uncertainties in the reddening correction and flux calibration, we have normalized all the spectra to $\mathrm{H} 11 \lambda 3770$. The resultant $T_{\mathrm{e}}(\mathrm{He} \mathrm{I})$ and $T_{\mathrm{e}}(\mathrm{H} \mathrm{I})$ are given in Table 1 . Given the relations $T_{\mathrm{e}}(\mathrm{He} \mathrm{I}, \mathrm{H} \mathrm{I}) \propto$ $J_{\mathrm{He}, \mathrm{H}}^{-\alpha}$, where $\alpha \approx 1.5$, the errors introduced by measurement uncertainties can be estimated through

$$
\Delta T_{\mathrm{e}}(\mathrm{He} \mathrm{I}, \mathrm{H} \mathrm{I})=\frac{3 \sigma}{J_{\mathrm{He}, \mathrm{H}}} T_{\mathrm{e}}(\mathrm{He} \mathrm{I}, \mathrm{H} \mathrm{I}),
$$

where $\sigma$ is the rms noise derived from the spectral regions free of emission lines, which cover a wide wavelength range. We found that for all the nebulae the $3 \sigma$ values are well below the $J_{\mathrm{He}, \mathrm{H}}$ values. Another error source arises in the uncertainties of the 


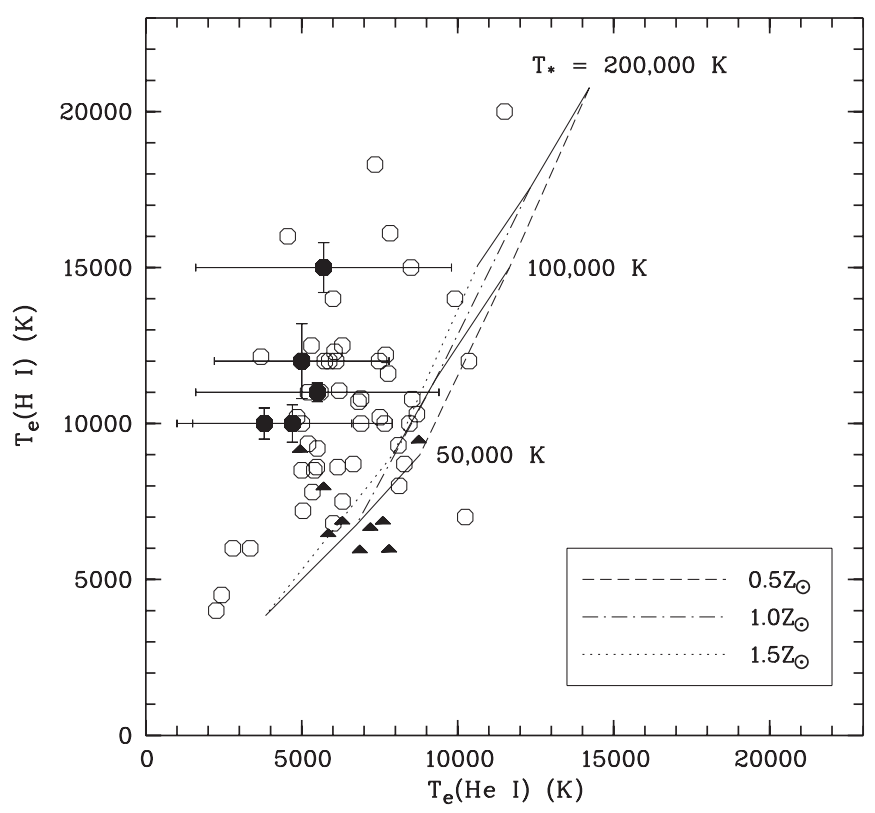

Figure 3. $T_{\mathrm{e}}(\mathrm{H} \mathrm{I})$ vs. $T_{\mathrm{e}}(\mathrm{He} \mathrm{I})$. The filled circles with error bars are those with $T_{\mathrm{e}}$ (He I) determined from the He I discontinuities in this work. The open circles are those with $T_{\mathrm{e}}(\mathrm{He} \mathrm{I})$ deduced from He I lines in Paper I. The filled triangles are the results for H II regions obtained by García-Rojas et al. (2005). These lines are the predictions by CLOUDY for different effective temperatures of the central stars and metallicities of the nebulae (see the text).

He I line emission coefficients, which might introduce an error in the He I temperature determination of about $20 \%$ according to a comparison of the atomic data given by different authors (Brocklehurst et al. 1999; Benjamin et al. 1999; Bauman et al. 2005). For high-excitation PNe, additional errors are caused by the strong $\mathrm{O}$ III Bowen lines around the He I discontinuities. In such cases, our fittings were based on the spectral regions with wavelengths departing from $3421 \AA$, introducing a $\sim 15 \%$ error in derived $T_{\mathrm{e}}(\mathrm{He} \mathrm{I})$ caused by uncertainties in flux calibration. The emission efficient of hydrogen two-photon continuity was deduced under Case A assumption and might be significantly underestimated under Case $\mathrm{B}$ conditions due to the collisional excitation from $2^{2} P$ to $2^{2} S$. However, the excess emission of the two-photon process can be mostly compensated by the simulated scattering continuities, and we do not require accurate estimate of the absolute magnitude of the two-photon emission. The uncertainties in the smooth continuities hardly affect our results mainly based on the $3421 \AA$ discontinuity.

The right panels of Figure 2 give zoom-in views of the spectra around the He I discontinuities. For some $\mathrm{PNe}$, the narrow spectral ranges from 3421 to $3500 \AA$ cannot be well fitted by our models due to the facts that the models did not treat the extended wings of $\mathrm{O}$ III Bowen lines and the blended high- $J$ He I lines at the red side of the $3421 \AA$ discontinuity, and the low sensitivity near the CCD edges leads to a larger slope. Nevertheless, these effects on our results should be somewhat insignificant since the deviations exist only in a relatively limited wavelength range. Of course, if our models merely take into account the spectra from 3300 to $3600 \AA$, we can achieve a better fitting in such a narrow wavelength range by adjusting the contribution from scattering continuities. However, this will result in a failed fitting of the global spectra. Modeling different spectral ranges to a reasonably good extent, we have assessed the errors in $T_{\mathrm{e}}(\mathrm{He} \mathrm{I})$. In Figure 2, we overlaid the best fitting to the narrow spectral ranges (3300-3600 $\AA$ ) of NGC 2440,
Table 2

He I Line Intensities Relative to $I(\mathrm{H} \beta)=1$ and $T_{\mathrm{e}}(\mathrm{He} \mathrm{I})$ Derived from He I Lines

\begin{tabular}{lccccc}
\hline \hline Transition & IC 2501 & IC 4634 & NGC 2440 & NGC 3242 & NGC 3918 \\
\hline Singlets & & & & & \\
$\lambda 6678$ & 0.0400 & 0.0590 & 0.0244 & 0.0381 & 0.0331 \\
$\lambda 7281$ & 0.0086 & 0.0142 & 0.0056 & 0.0082 & 0.0068 \\
Triplets & & & & & \\
$\lambda 5876$ & 0.1790 & 0.2125 & 0.1039 & 0.1210 & 0.1367 \\
$\lambda 7065$ & 0.0904 & 0.1057 & 0.0393 & 0.0494 & 0.0513 \\
$T_{\mathrm{e}}(\mathrm{He} \mathrm{I})^{\mathrm{a}}$ & $7500 \mathrm{~K}$ & $8400 \mathrm{~K}$ & $8000 \mathrm{~K}$ & $7400 \mathrm{~K}$ & $7100 \mathrm{~K}$ \\
\hline
\end{tabular}

Note.

${ }^{\text {a }}$ Bears an error of $\sim 2200 \mathrm{~K}$.

NGC 3242, and NGC 3918, which results in $T_{\mathrm{e}}(\mathrm{He} \mathrm{I})=$ $6840 \mathrm{~K}, 7500 \mathrm{~K}$, and $8250 \mathrm{~K}$ for the three high-excitation PNe, respectively. We noted that these values, which should be larger than the real $T_{\mathrm{e}}\left(\mathrm{He}_{\mathrm{I}}\right)$ values, are still lower than $T_{\mathrm{e}}(\mathrm{H} \mathrm{I})$.

Three of the PNe in the recent sample have been studied in Paper I. For comparison, Table 1 gives $T_{\mathrm{e}}(\mathrm{H} \mathrm{I})$ and $T_{\mathrm{e}}(\mathrm{He} \mathrm{I})$ determined in Paper I. Considering the error bars, they are in agreement with the results derived in the recent work. Note that the $T_{\mathrm{e}}(\mathrm{He} \mathrm{I})$ in Paper I were derived from $\mathrm{He} \mathrm{I}$ lines ratios and might possibly be different from those derived from He I discontinuities due to the effects of radiative transfer and temperature inhomogeneities.

In Figure 3, we compare $T_{\mathrm{e}}(\mathrm{H} \mathrm{I})$ and $T_{\mathrm{e}}(\mathrm{He} \mathrm{I})$. These points derived in the recent work and those derived in Paper I are located at approximately the same position in this figure. An inspection of Figure 3 shows that $T_{\mathrm{e}}(\mathrm{He} \mathrm{I})$ is consistently lower than $T_{\mathrm{e}}(\mathrm{H} \mathrm{I})$, confirming our previous results in Paper I. In the scenario of the two-abundance model, the He I emission is significantly contaminated by emission from the extremely cold hydrogen-deficient inclusions, while the $\mathrm{H}$ I recombination spectra dominantly originate in the normal component under a typical temperature of $T_{\mathrm{e}} \sim 10^{4} \mathrm{~K}$. This provides a plausible explanation for the lower $T_{\mathrm{e}}(\mathrm{He} \mathrm{I})$ compared to $T_{\mathrm{e}}(\mathrm{H} \mathrm{I})$. In Figure 3 , we also overplot the results for $\mathrm{H}$ II regions obtained by García-Rojas et al. (2005), which are located in a more compact area in this figure and indicate to a smaller $T_{\mathrm{e}}(\mathrm{He} \mathrm{I}) / T_{\mathrm{e}}(\mathrm{H} \mathrm{I})$ discrepancy in $\mathrm{H}$ II regions compared to $\mathrm{PNe}$.

\section{DISCUSSION}

\subsection{He I Lines}

A few strong He I lines have been clearly detected in the red arm. They allow an independent examination of our results derived from the He I discontinuities. This is nontrivial in that density inhomogeneities and radiative transfer effects could affect our results to some extent.

Here, we took into account the four strongest He I lines in the red arm, $\lambda \lambda 7281,6678,7065$, and 5876, as shown in Figure 4. Their intensities are listed in Table 2. In Paper I, we have shown that the He I ratio $I(\lambda 7281) / I(\lambda 6678)$ provides an useful temperature diagnostic. According to Benjamin et al. (2002), the He I $\lambda \lambda 7065,5876$ lines are from triplet states and are essentially affected by the optical depth of the $2 s^{3} S$ level. Figure 5 gives the theoretical predictions of the $\mathrm{He}$ I ratio $I(\lambda 7281) / I(\lambda 6678)$ versus He I ratio $I(\lambda 7065) / I(\lambda 5876)$ at different electron temperatures, electron densities $\left(N_{\mathrm{e}}\right)$, and optical depths of the He I $2 s^{3} S-3 p^{3} P^{\circ} \lambda 3889$ line $\left(\tau_{3889}\right)$. The observed values for our sample are also shown in this figure. 

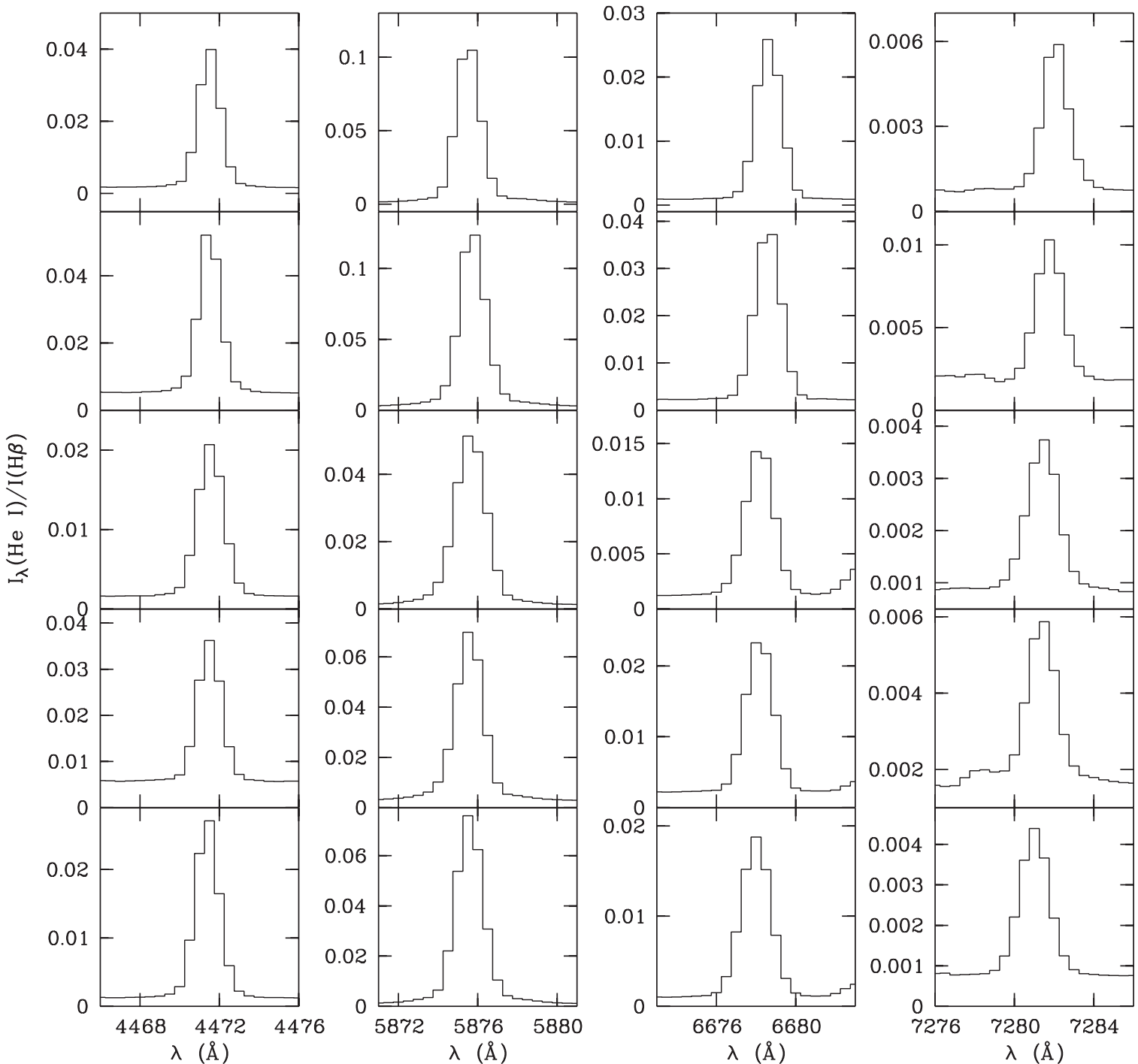

Figure 4. He I 4471, 5876, 6678, 7281 lines (from left to right) detected in IC 2501, IC 4634, NGC 2240, NGC 3242, and NGC 3918 (from top to bottom).

The diagram is useful to investigate nebular physical conditions. An inspection of Figure 5 shows that the $T_{\mathrm{e}}(\mathrm{He} \mathrm{I})$ values are consistently lower than $10,000 \mathrm{~K}$, which represents the lowest value of $T_{\mathrm{e}}(\mathrm{H} \mathrm{I})$ in our sample, supporting the conclusion drawn from the He I discontinuities. We also found that the $\tau_{3889}$ values range from 0 to 10 . These line ratios have only weak dependence on electron densities and are hardly used to determined $N_{\mathrm{e}}(\mathrm{He}$ I).

Table 2 gives the $T_{\mathrm{e}}(\mathrm{He} \mathrm{I})$ values derived from the $\mathrm{He} \mathrm{I}$ line observations. The uncertainties of electron densities and optical opacities introduce an error of about $1500 \mathrm{~K}$. Using the spectral lines falling in the overlapping wavelength region of two adjacent orders, we estimated the uncertainties in flux calibration to be about $5 \%$. The combined errors of the resultant $T_{\mathrm{e}}(\mathrm{He} \mathrm{I})$ amounts to about $2200 \mathrm{~K}$. The $T_{\mathrm{e}}(\mathrm{He} \mathrm{I})$ deduced in this paper are biased to larger values compared to those in Paper I (although they are marginally consistent with each other considering the error bars). This is partly attributed to the fact that in the present observation the slit was put in a position closer to the center. However, the primary cause might be that the uncertainties in flux calibration of the red arm are probably larger than expect. We performed a comparison of the present spectra and our previous long-slit spectra and found that the former have systematically higher line fluxes in very red wavelength regions. Therefore, we suppose that the fluxes of the He I $\lambda 7281$ might have been significantly overestimated, and the $T_{\mathrm{e}}(\mathrm{He} \mathrm{I})$ in Table 2 should be treated as upper limits, which are still lower than $T_{\mathrm{e}}(\mathrm{H} \mathrm{I})$. A detailed discussion of $\mathrm{He}$ I lines requires more precise observations in the red arm and is beyond the focus of the present paper.

One should bear in mind that the electron temperatures derived from the $\mathrm{He}$ I discontinuities and those from the $\mathrm{He}$ I line ratios are not necessary identical since they have different temperature dependences, and thus weight different regions if large temperature fluctuations exist. The comparison of $\mathrm{He} \mathrm{I}$ discontinuity and line temperatures is a potentially intriguing subject for future research.

\subsection{Model Tests}

An issue is invited, whether the large $T_{\mathrm{e}}(\mathrm{HI}) / T_{\mathrm{e}}(\mathrm{He} \mathrm{I})$ discrepancies in high-excitation PNe can be ascribed to the presence of substantial $\mathrm{He}^{2+}$ zone which has a higher temperature than $\mathrm{He}^{+}$ zone. In order to investigate the problem, we first compare the observed results with the predictions by one-dimensional photoionization models of chemically homogeneous nebulae. We 


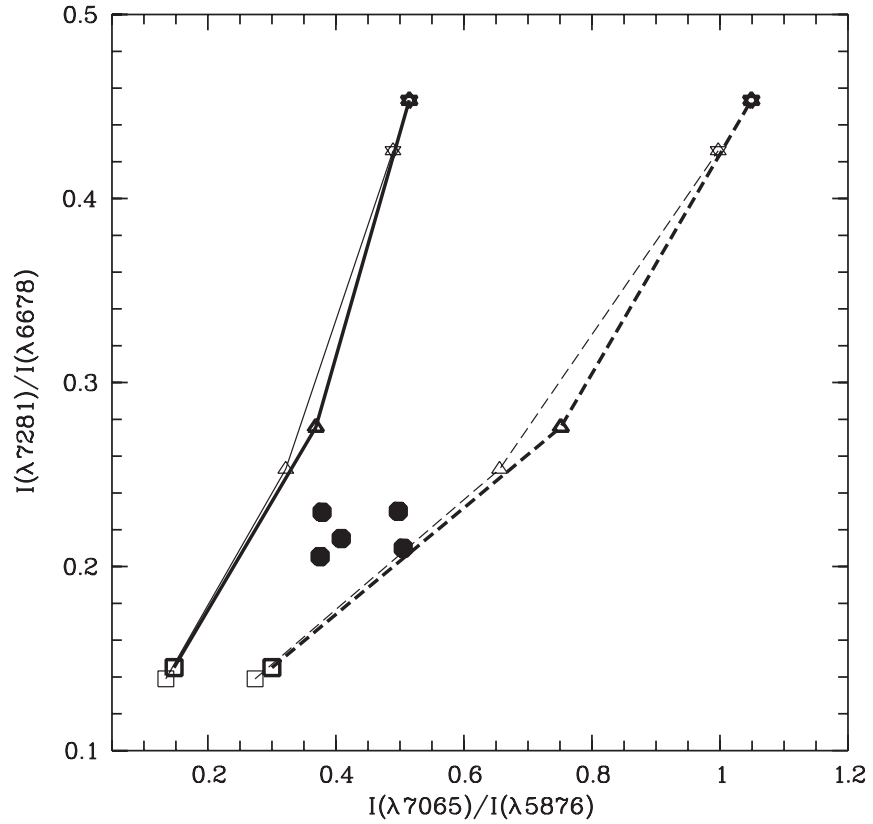

Figure 5. Theoretical predictions of the He I line ratios $I(\lambda 7281) / I(\lambda 6678)$ vs. $I(\lambda 7065) / I(\lambda 5876)$, derived at different physical conditions: $T_{\mathrm{e}}=5000 \mathrm{~K}$ (squares), $10,000 \mathrm{~K}$ (triangles), and 20,000, K (stars); $N_{\mathrm{e}}=10^{4} \mathrm{~cm}^{-3}$ (light lines) and $10^{6} \mathrm{~cm}^{-3}$ (thick lines); $\tau_{3889}=0$ (solid lines) and 10 (dashed lines). The filled circles represent the observations.

then construct three-dimensional photoionization models to test the two-abundance model as the explanation of the temperature discrepancies.

\subsubsection{Chemically Homogeneous Model}

To date, the published photoionization models for certain $\mathrm{PNe}$ with a chemically homogeneous medium predict only minor discrepancy between $T_{\mathrm{e}}(\mathrm{He} \mathrm{I})$ and $T_{\mathrm{e}}(\mathrm{H} \mathrm{I})$. For example, the classic photoionization model of NGC 7662 constructed by Harrington et al. (1982) yields $T_{\mathrm{e}}(\mathrm{H} \mathrm{I})-T_{\mathrm{e}}(\mathrm{He} \mathrm{I}) \approx 600 \mathrm{~K}$. To investigate the case of general PNe, we use the CLOUDY code (Ferland et al. 1998) to construct a series of one-dimensional photoionization modeling of chemically homogeneous nebulae. In order to compare the modeling results with the observed ones, we define modeled $T_{\mathrm{e}}(\mathrm{He} \mathrm{I})$ and $T_{\mathrm{e}}(\mathrm{H} \mathrm{I})$ by

$$
\begin{aligned}
T_{\mathrm{e}}(\mathrm{He} \mathrm{I}, \mathrm{H} \mathrm{I}) & =\frac{\int T_{\mathrm{e}}(r) J_{\mathrm{He}, \mathrm{H}}(r) d r}{\int J_{\mathrm{He}, \mathrm{H}}(r) d r} \\
& \approx \frac{\int T_{\mathrm{e}}(r) N_{\mathrm{e}}(r) N_{\mathrm{He}^{+}, \mathrm{H}^{+}}(r) T_{\mathrm{e}}(r)^{-2 / 3} d r}{\int N_{\mathrm{e}}(r) N_{\mathrm{He}^{+}, \mathrm{H}^{+}}(r) T_{\mathrm{e}}(r)^{-2 / 3} d r},
\end{aligned}
$$

where $r$ is the position vector, $N_{\mathrm{e}}(r)$ is the electron density, and $N_{\mathrm{He}^{+}, \mathrm{H}^{+}}(r)$ is the number density of $\mathrm{He}^{+}$or $\mathrm{H}^{+}$atoms. Then $T_{\mathrm{e}}(\mathrm{He} \mathrm{I})$ and $T_{\mathrm{e}}(\mathrm{H} \mathrm{I})$ can be simulated from the modeled $T_{\mathrm{e}}(r)$, $N_{\mathrm{e}}(r)$, and $N_{\mathrm{He}^{+}, \mathrm{H}^{+}}(r)$. The temperatures defined in Equation (2) are similar to those derived from the observed $\mathrm{He}$ I and $\mathrm{H}$ I discontinuities.

For the modeling, a blackbody spectral energy distribution with a luminosity $L_{*}=10^{38} \mathrm{erg} \mathrm{s}^{-1}$ is assumed for the central star. We assume that the nebula has a homogeneous density structure with a hydrogen density of $n(\mathrm{H})=10^{3} \mathrm{~cm}^{-3}$. The assumed $n(\mathrm{H})$ represents the lower limit of typical PNe. At a given UV radiation field, increasing $n(\mathrm{H})$ will decrease the ionization parameter and the $\mathrm{He}^{2+} / \mathrm{He}^{+}$ratio, and thus decrease the contribution of $\mathrm{He}^{2+}$ zone to the $T_{\mathrm{e}}(\mathrm{He} \mathrm{I}) / T_{\mathrm{e}}(\mathrm{H} \mathrm{I})$ discrepancies.
Our modeling reproduces the $T_{\mathrm{e}}(\mathrm{He} \mathrm{I})$ and $T_{\mathrm{e}}(\mathrm{H} \mathrm{I})$ values in different nebular metallicities $(Z)$ and effective temperature of the ionizing $\operatorname{star}\left(T_{*}\right)$. The results are illustrated in Figure 3. The modeling shows that the presence of $\mathrm{He}^{2+}$ zone can cause a higher $T_{\mathrm{e}}(\mathrm{H} \mathrm{I})$ compared to $T_{\mathrm{e}}(\mathrm{He} \mathrm{I})$. The $T_{\mathrm{e}}(\mathrm{H}$ I) $/ T_{\mathrm{e}}$ (He I) discrepancies sharply increase with increasing $T_{*}$ and have only a weak dependency on $Z$. Nevertheless, the large differences between the observed $T_{\mathrm{e}}(\mathrm{He} \mathrm{I})$ and $T_{\mathrm{e}}(\mathrm{H}$ I) are obviously beyond the model predictions although the claim is probably not applied for H II regions. An inspection of Figure 3 suggests that chemically homogeneous models are unable to reproduce the extremely low $T_{\mathrm{e}}(\mathrm{He} \mathrm{I})$ measured in PNe. The observed $T_{\mathrm{e}}(\mathrm{H}$ I) values can be well reproduced by using appropriate $T_{*}$ and $Z$. However, in order to match the observed $T_{\mathrm{e}}(\mathrm{He} \mathrm{I})$ for most $\mathrm{PNe}$, we require extremely high $T_{*}$ $(\gg 200,000 \mathrm{~K})$ and $Z\left(\gg 1.5 Z_{\odot}\right)$, which are unlikely real for our sample.

Our models do not include the photoelectric heating by dust grains, which can efficiently enhance the temperature in the nebular regions close to the central star (Stasińska \& Szczerba 2001). In addition, there might be other unexpected heating sources that lead to greater temperature fluctuations. It is too early to conclude with certainty that chemical inhomogeneities must be introduced to explain the observed temperature discrepancies. In any case, a more comprehensive photoionization model including extra heating mechanisms should take into account the He I discontinuities.

\subsubsection{The Two-Abundance Model}

To test the two-abundance model, we use the threedimensional photoionization code MOCASSIN (Ercolano et al. 2003a), which is capable of treating nebular geometric asymmetries, density and composition inhomogeneities as well as the diffuse radiation fields self-consistently, and thus enables to more rigorously study the physical properties and chemical composition of the postulated hydrogen-deficient component compared to the one-dimensional model. A series of two-abundance models are constructed and the reproduced $J_{\mathrm{H}}$ and $J_{\mathrm{He}}$ are compared with the observed values. We found that the presence of a very small amount of hydrogen-deficient material is enough to interpret the observations. An example is given in Figure 6, where we compare the normalized $J_{\mathrm{H}}$ and $J_{\mathrm{He}}$ observed for our PN sample and those predicted by one of the MOCASSIN models. For this two-abundance model, we have assumed that the diffuse nebula has a spherical structure, an $n(\mathrm{H})$ value of $10^{3} \mathrm{~cm}^{-3}$, and a metallicity of $Z_{\mathrm{PN}}$, where $Z_{\mathrm{PN}}$ is the average metallicity of the Galactic PNe (Kingsburgh \& Barlow 1994). The hydrogendeficient inclusions have a filling factor of $0.18 \%$ and an $n(\mathrm{H})$ value of $70 \mathrm{~cm}^{-3}$, and are enhanced by a factor of 200 in $\mathrm{He}$ and by a factor of 800 in CNONe compared to the diffuse gas. An effective temperature of $120,000 \mathrm{~K}$ is assumed for the central star. According to our modeling, the electron temperature of the hydrogen-deficient inclusions is about $500 \mathrm{~K}$, which is much lower than the average temperature of the diffuse gas of $10,770 \mathrm{~K}$. The cold hydrogen-deficient inclusions contribute about $45 \%$ and $0.2 \%$ of $\mathrm{He} \mathrm{I}$ and $\mathrm{H} \mathrm{I}$ line emission intensities, respectively. As shown in Figure 6, the model result closely matches the observed $J_{\mathrm{H}}$ and $J_{\mathrm{He}}$.

For comparison, we construct a series of chemically homogeneous models using MOCASSIN in the parameter ranges $50,000 \mathrm{~K}<T_{*}<200,000 \mathrm{~K}, 500 \mathrm{~cm}^{-3}<n(\mathrm{H})<8000 \mathrm{~cm}^{-3}$, and $0.6 Z_{\mathrm{PN}}<Z<1.6 Z_{\mathrm{PN}}$. The predicted region of $J_{\mathrm{H}}$ and 


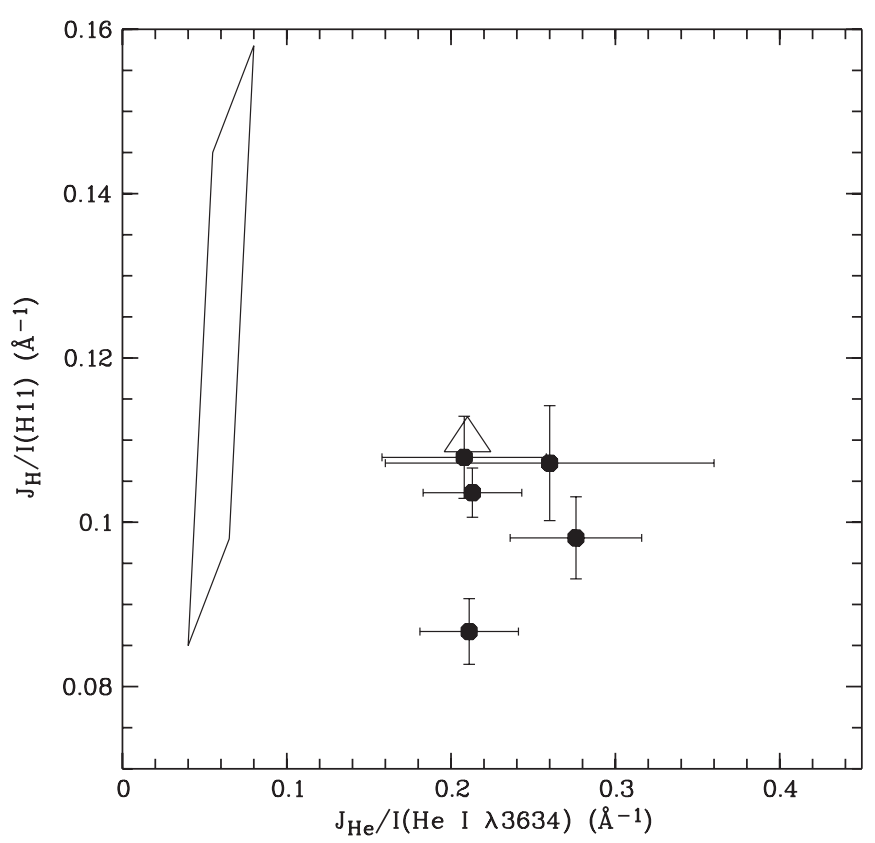

Figure 6. $J_{\mathrm{H}} / I(\mathrm{H} 11)$ vs. $J_{\mathrm{He}} / I\left(\mathrm{He}_{\mathrm{I}} \lambda 3634\right)$. The filled circles denote the observed values. The open triangle is the prediction of a two-abundance model (see the text). The enclosed region represents the zone occupied by the predictions of chemically homogeneous models by MOCASSIN in the parameter ranges $50,000 \mathrm{~K}<T_{*}<200,000 \mathrm{~K}, 500 \mathrm{~cm}^{-3}<n(\mathrm{H})<8000 \mathrm{~cm}^{-3}$, and $0.6 Z_{\mathrm{PN}}<Z<1.6 Z_{\mathrm{PN}}$.

$J_{\mathrm{He}}$ is given in Figure 6. Again it is confirmed that even for high-excitation PNe, chemically homogeneous models are unable to reproduce the observed $\mathrm{He}$ I discontinuities.

The two-abundance model can provide a natural solution for the observed $T_{\mathrm{e}}(\mathrm{He} \mathrm{I}) / T_{\mathrm{e}}(\mathrm{H} \mathrm{I})$ discrepancies. Nevertheless, we have noted some problems in our two-abundance modeling. In order to explain the low $T_{\mathrm{e}}(\mathrm{He} \mathrm{I})$, we require to assume a low density and high enhancement factor of heavy elements for the hydrogen-deficient inclusions to get effective cooling. Such a low-density and low-temperature component is unstable inside the nebulae. Moreover, our models yield an ADF of 1020, higher than the typical value for general PNe. Probably, these imply to the presence of some additional coolants inside hydrogen-deficient inclusions.

The hydrogen-deficient inclusions postulated in the current study have similar properties to Abell 30's knots, which show extremely He-rich (Wesson et al. 2003; Ercolano et al. 2003b). However, the properties of the hydrogen-deficient inclusions in different PNe are probably not identical. A complete understanding of the hydrogen-deficient component requires detailed modeling and precise measurements of heavy-element ORLs for individual $\mathrm{PNe}$, which is beyond the scope of this paper. Our results show that the observations of $\mathrm{He}$ I discontinuities can provide some constraints on future study of the hydrogendeficient inclusions.

\section{CONCLUSIONS}

Based on a high signal-to-noise spectroscopy of a sample of $\mathrm{PNe}$, we explored the possibility of using the He I discontinuities at $3646 \AA$ as probe of nebular physical conditions. $T_{\mathrm{e}}(\mathrm{He} \mathrm{I})$ is found to be systematically lower than $T_{\mathrm{e}}(\mathrm{H} \mathrm{I})$, confirming the results of Paper I, in which we determined $T_{\mathrm{e}}(\mathrm{He}$ I) using the $\mathrm{He}$ I recombination lines. We have investigated the contribution of $\mathrm{He}^{2+}$ zone on the $T_{\mathrm{e}}(\mathrm{He} \mathrm{I}) / T_{\mathrm{e}}(\mathrm{H} \mathrm{I})$ discrepancies by constructing a series of photoionization models. We have shown that the chemically homogeneous models predict too small temperature discrepancies as compared to the observations. The observations, however, can be naturally interpreted if one assumes the presence of a small amount of hydrogendeficient cold inclusions within the diffuse nebula. Our results thus provide a possible evidence in favor of the two-abundance model as the explanation of the CEL/ORL abundance problem. Nevertheless, we cannot completely rule out the scenario of temperature fluctuations if some extra heating mechanisms exist.

To completely understand the properties of the postulated small hydrogen-deficient inclusions, we need to construct detailed three-dimensional photoionization models with very fine mesh grids and take account of the ORLs from helium and heavy elements. For that, an enormous amount of computer time is required. The detailed two-abundance models for individual objects will be reported in forthcoming papers (e.g., H.-B. Yuan et al. 2009, in preparation).

In the current paper, we show that the He I discontinuities, not withstanding some observational and technical limitations, provide a valuable probe of nebular physical conditions and can place a tight constraint for the modeling. The method proposed in this paper is particularly appropriate for studying low-excitation $\mathrm{PNe}$ and $\mathrm{H}$ II regions that are free from the effects of strong O III Bowen lines. Instrument development and atomic data improvement will be invaluable in providing more robust conclusions.

The authors are grateful to M. A. Dopita and B. RoccaVolmerange for their help in ensuring successful observations. We thank the anonymous referee for useful comments. C.T.H. is grateful to the University of Hong Kong (HKU) for having made the present output possible. The observations were made in the frame of the Programme International de Coopération Scientifique PICS France-Australie. The three-dimensional modeling was carried out on the SGI Altix330 System at Department of Astronomy, Peking University and the HPCPOWER cluster of HKU. J.N. acknowledges financial support from Seed Funding Programme for Basic Research in HKU (200802159006). The work was partially supported by the Research Grants Council of Hong Kong under grants HKU7028/07P and HKU7033/08P.

\section{REFERENCES}

Bauman, R. P., Porter, R. L., Ferland, G. J., \& MacAdam, K. B. 2005, ApJ, 628, 541

Benjamin, R. A., Skillman, E. D., \& Smits, D. P. 1999, ApJ, 514, 307

Benjamin, R. A., Skillman, E. D., \& Smits, D. P. 2002, ApJ, 569, 288

Brocklehurst, M. 1972, MNRAS, 157, 21

Cahn, J. H., Kaler, J. B., \& Stanghellini, L. 1992, A\&AS, 94, 399

Ercolano, B., Barlow, M. J., Storey, P. J., \& Liu, X.-W. 2003a, MNRAS, 340, 1136

Ercolano, B., Barlow, M. J., Storey, P. J., Liu, X.-W., Rauch, T., \& Werner, K. 2003b, MNRAS, 344, 1145

Ercolano, B., \& Storey, P. J. 2006, MNRAS, 372, 1875

Ferland, G. J., Korista, K. T., Verner, D. A., Ferguson, J. W., Kingdon, J. B., \& Verner, E. M. 1998, PASP, 110, 761

García-Rojas, J., Esteban, C., Peimbert, A., Peimbert, M., Rodríguez, M., \& Ruiz, M. T. 2005, MNRAS, 362, 301

Guseva, N. G., Izotov, Y. I., Papaderos, P., \& Fricke, K. J. 2007, A\&A, 464, 885

Guseva, N. G., Izotov, Y. I., \& Thuan, T. X. 2006, ApJ, 644, 890

Harrington, J. P., Seaton, M. J., Adams, S., \& Lutz, J. H. 1982, MNRAS, 199, 517

Howarth, I. D. 1983, MNRAS, 203, 301 
Kingsburgh, R. L., \& Barlow, M. J. 1994, MNRAS, 271, 257

Liu, X.-W. 2006, in IAU Symp. 234, Planetary Nebulae, ed. M. J. Barlow \& R. H. Méndez (Cambridge: Cambridge Univ. Press), 219

Liu, X.-W., Barlow, M. J., Zhang, Y., Bastin, R. J., \& Storey, P. J. 2006, MNRAS, 368,1959

Liu, X.-W., \& Danziger, I. J. 1993, MNRAS, 263, 256

Peimbert, M. 1967, ApJ, 150, 825

Peimbert, M. 1971, Bol. Obs. Tonantzintla Tacubaya, 6, 29
Peimbert, M., \& Peimbert, A. 2006, in IAU Symp. 234, Planetary Nebulae, ed. M. J. Barlow \& R. H. Méndez (Cambridge: Cambridge Univ. Press), 227 Stasińska, G., \& Szczerba, R. 2001, A\&A, 379, 1024

Wesson, R., Liu, X.-W., \& Barlow, M. J. 2003, MNRAS, 340, 253

Zhang, Y., Liu, X.-W., Liu, Y., \& Rubin, R. H. 2005a, MNRAS, 358, 457 (Paper I)

Zhang, Y., Rubin, R. H., \& Liu, X.-W. 2005b, RevMexAA, 23, 15

Zhang, Y., et al. 2004, MNRAS, 351, 935 